\title{
Voraussetzungen und Qualitätskriterien von Mixed-Methods-Studien in der mathematikdidaktischen Forschung
}

\author{
Nils Buchholtz iD
}

Eingegangen: 13. April 2019 / Angenommen: 18. August 2020 / Online publiziert: 16. September 2020 (C) Der/die Autor(en) 2020

Zusammenfassung Die Verbreitung von Mixed-Methods-Studien in der Mathematikdidaktik wirft die Frage nach disziplinspezifischen Voraussetzungen und Qualitätskriterien auf. Derartige Studien sollten daher nicht nur pragmatisch oder durch die Auswahl von spezifischen Forschungsdesigns begründet werden, sondern auch den gegenstandsspezifischen Mehrwert einer Integration von Ergebnissen herausstellen, die durch unterschiedliche (mathematikdidaktische) Forschungsmethoden gewonnen wurden. Im Beitrag werden deshalb methodologische und wissenschaftsphilosophische Voraussetzungen zu Mixed-Methods-Studien ontologischen und epistemologischen Annahmen zu mathematikdidaktischen Forschungsgegenständen und Methoden gegenübergestellt. Eine theoretische Verankerung von Mixed-MethodsStudien im Critical Realism ermöglicht, die Kombination unterschiedlicher Forschungsparadigmen auch ontologisch zu begründen. Die aus den disziplinspezifischen Anknüpfungspunkten entwickelte Perspektive für Qualitätskriterien und ein methodologisches Framework kann zu einer reflektierten Praxis von Mixed-Methods in der Mathematikdidaktik beitragen

Schlüsselwörter Mixed-Methods-Forschung · Forschungsmethoden · Qualitative Methoden · Quantitative Methoden · Stoffdidaktik

\section{Requirements and quality criteria of mixed method studies in mathematics education research}

Abstract The prevalence of mixed method studies in mathematics education raises the question of discipline-specific requirements and quality criteria. Such studies

\footnotetext{
N. Buchholtz $(\bowtie)$

Department of Teacher Education and School Research, University of Oslo, 1099, 0317 Oslo,

Norway

E-Mail: N.F.Buchholtz@ils.uio.no
} 
should therefore not only be motivated pragmatically or by the selection of specific research designs, but should also emphasize the subject-specific added value of integrating results obtained by different research methods (of mathematics education). Therefore, the article contrasts methodological and scientific-philosophical prerequisites for mixed-method studies with ontological and epistemological assumptions about research objects and methods in mathematics education. A theoretical anchoring of mixed method studies in Critical Realism enables to justify the combination of different research paradigms also from an ontological point of view. The perspective for quality criteria and a methodological framework developed from the discipline-specific starting points can contribute to a reflected practice of mixed methods research in mathematics education.

Keywords Mixed methods research · Research methodology · Qualitative methods $\cdot$ Quantitative methods $\cdot$ Subject-matter didactics

\section{Einleitung}

Die Anzahl der Studien, die sowohl von qualitativen als auch von quantitativen Forschungsmethoden Gebrauch machen, hat in den letzten Jahren in der deutschsprachigen mathematikdidaktischen Forschung zugenommen (siehe etwa Lüken und Sauzet 2020; Schüler-Meyer et al. 2017; Reindl 2016; Hoth 2016; Schulz 2010). Trotz einer Reihe von Beispielen lässt sich der Anteil an Studien, die sich der Mixed-Methods-Forschung (MMF) zuordnen lassen, insgesamt nur schwer beziffern, weil sich sowohl voll integrierte Mixed-Methods-Studien als auch qualitative und quantitative Einzelstudien ausmachen lassen, die erst im Zusammenhang in ein größeres Mixed-Methods-Forschungsvorhaben eingehen. Zumindest konnten sogenannte Mixed-Methods-Prävalenz-Studien von Hart et al. (2009) oder Ross und Onwuegbuzie (2012) aber zeigen, dass in $29 \%$ bzw. $31 \%$ der in ausgewählten internationalen mathematikdidaktischen Journalen publizierten Artikel Gebrauch von sowohl qualitativen als auch quantitativen Methoden gemacht wird (vgl. Buchholtz 2019). Einen ähnlichen Anteil stellen Riazi und Candlin (2014) auch für den Bereich der Sprachdidaktik fest. Aufgrund dieser Relevanz sollten Voraussetzungen und Qualitätskriterien derartiger Studien in der Mathematikdidaktik genauer diskutiert werden.

Allgemeine Qualitätskriterien von Mixed-Methods-Studien sind u. a. das Vorhandensein einer Begründung für den Forschungsansatz und eine realisierte Integration von Forschungsergebnissen, die durch Kombination oder Integration unterschiedlicher Methoden im Forschungsprozess ermittelt wurden (vgl. Schoonenboom und Johnson 2017; Mertens et al. 2016; vgl. auch Abschn. 2). Diese Kriterien sind jedoch nicht immer gegeben. So unterzog Bryman (2008) 232 empirische Mixed-MethodsArtikel aus unterschiedlichen Bereichen der Sozialwissenschaften einer genaueren Inhaltsanalyse. Dabei konnte nur in $53 \%$ der Fälle eine Integration von Forschungsergebnissen ausgemacht werden, während bei nahezu der Hälfte der Artikel eine rein parallele, oft nicht aufeinander bezogene Darstellung der unterschiedlichen Forschungsergebnisse vorliegt. Weitere Qualitätsprobleme stellt Riazi (2016) für Mixed- 
Methods-Studien in der Sprachdidaktik fest, in denen die Verwendung unterschiedlicher Methoden oft rein pragmatisch und ,technisch orientiert“ (S. 34) an gewählten Forschungsdesigns erfolgt. Die Forschungsfragen werden dabei nur unzureichend theoretisch in Bezug auf die Natur des Forschungsgegenstandes verankert, und der Gebrauch von sowohl qualitativen als auch quantitativen Methoden wird nicht ausreichend methodologisch gerechtfertigt. Zwar stehen derartige Analysen für MixedMethods-Studien speziell in der Mathematikdidaktik derzeit noch aus, aber die Gefahr einer unzureichenden Begründung, einer fehlenden theoretischen Verankerung oder einer nicht ausreichend vorgenommenen Integration von Ergebnissen ist auch hier gegeben.

Die Begründung der Wahl eines Mixed-Methods-Forschungsansatzes ist wesentlicher Bestandteil eines Arguments für die Validität der Schlussfolgerungen, die mit den eingesetzten Methoden generiert werden können (Newton und Shaw 2014). Für Mixed-Methods-Studien ist dabei klassischerweise abzuwägen, inwieweit eine gemischt-methodische Herangehensweise Vorteile gegenüber mono-methodisch orientierten Vorgehen beinhaltet. Dies hängt mit einer auf den Forschungsgegenstand gerichteten Reflexion darüber zusammen, welche spezifischen Stärken und Schwächen qualitative und quantitative Methoden aufweisen, und inwieweit ein gemischtmethodisches Vorgehen diese Stärken vereinen bzw. die Schwächen ausgleichen kann (Johnson und Christensen 2017). Dabei ist auch zu beachten, dass MixedMethods-Studien Mono-Method-Studien nicht prinzipiell überlegen sind, also die Integration von durch unterschiedliche Forschungsmethoden generierten Ergebnissen für eine Rechtfertigung einen erkenntnistheoretischen Mehrwert in Bezug auf den Forschungsgegenstand hervorbringen sollte. Damit berührt das Kriterium der Begründung allerdings nicht nur allgemein-methodische, sondern auch disziplinspezifische Überlegungen.

Disziplinspezifische Qualitätskriterien von Mixed-Methods-Studien betreffen damit einerseits an mathematikdidaktischen Forschungsgegenständen orientierte Begründungen und andererseits spezifische methodologische Annahmen mathematikdidaktischer Forschung. Methodologisch ist etwa bislang noch nicht hinreichend geklärt, inwieweit sich mathematikdidaktische Forschungsmethoden wie die Stoffdidaktik im Rahmen von Mixed-Methods-Studien mit empirisch-sozialwissenschaftlichen Methoden verknüpfen lassen. Das Ziel dieses Artikels ist, einen Beitrag zur methodologischen Reflexion und Qualitätssicherung von MMF in der mathematikdidaktischen Forschung zu leisten. Die Ausführungen stellen eine Vertiefung der Überlegungen zu disziplinspezifischen Besonderheiten von MMF dar, die erstmalig in Buchholtz (2019) beschrieben wurden. ${ }^{1}$ Im Zentrum steht die Frage, welche methodologischen Voraussetzungen und Qualitätskriterien für die Durchführung von MMF in der Mathematikdidaktik sich aus dem disziplinären Zusammenhang erschließen lassen und von mathematikdidaktischen Mixed-Methods-Studien adres-

\footnotetext{
1 Die Publikation Buchholtz (2019) stellt eine Anleitung für (Nachwuchs)-Wissenschaftler in der Mathematikdidaktik dar, die ein Mixed-Methods-Forschungsvorhaben planen. Darin werden fachspezifische Besonderheiten des Ansatzes in der Mathematikdidaktik diskutiert. Der hier vorliegende Artikel folgt in wesentlichen Teilen den Überlegungen von Buchholtz (2019), vertieft diese aber erstens für eine deutsche Leserschaft und zweitens in Hinblick auf die methodologischen und wissenschaftsphilosophischen Voraussetzungen der MMF und auf die entsprechenden Annahmen mathematikdidaktischer MMF.
} 
siert werden sollten. Der Beitrag greift damit zum einen eine aktuelle methodologische Diskussion innerhalb der Mixed-Methods-Community auf und diskutiert zum anderen den Gebrauch von unterschiedlichen Forschungsmethoden innerhalb der Mathematikdidaktik.

\section{Begriffliche Grundlagen der MMF}

Mehrheitlich unterliegt dem Begriff Mixed Methods das Verständnis von einer Integration qualitativer und quantitativer Forschungsansätze im Sinne einer Methodentriangulation. Triangulation, ursprünglich ein der Geodäsie und Navigation entlehnter und in der quantitativen Methodologie beheimateter Begriff, wurde von Denzin (1978) als Bezeichnung für ein methodologisches Konzept zur Stärkung der Validität von Forschungsergebnissen eingeführt, wobei Denzin zwischen Theorien-, Daten-, Methoden- und Beobachtertriangulation unterschiedet. Innerhalb der Methodentriangulation war es dabei auch möglich, qualitative und quantitative Methoden aufeinander zu beziehen und die „Methoden gegeneinander auszuspielen, um die Validität der gewonnenen Ergebnisse zu maximieren“ (Denzin 1978, S. 304, eigene Übersetzung). Dieser Interpretation von Triangulation wurde jedoch entgegengehalten, dass unterschiedliche Methoden unterschiedliche theoretische und epistemologische Hintergrundannahmen und Traditionen mit sich bringen, so dass Ergebnisse nicht ohne weiteres vergleichbar sind und eine Validierung damit nicht immer gewährleistet ist (Kelle 2017). Von Flick (1998, S. 230) wurde daher vorgeschlagen, unter Triangulation auch die Stärkung der Tiefe und Breite von Forschungsergebnissen im Sinne einer Komplementarität zu verstehen. Seither ist Triangulation als Einnahme unterschiedlicher Perspektiven auch als Prinzip der qualitativen Forschung stark verbreitet. Dieses nicht mehr nur auf Validierung beschränkte Verständnis ist insbesondere in sozialwissenschaftlichen Kontexten relevant und bildet die argumentative Grundlage zur Untersuchung komplexer Forschungsgegenstände mit unterschiedlichen Methoden (Creswell und Plano Clark 2018). Kelle (2017) weist aus diesem Grund bei der methodologischen Beschreibung der MMF auf diese zwei Lesarten des Triangulationskonzepts hin und unterscheidet zwischen Methodenkombination (mit den Zielen Komplementarität der Befunde und ganzheitliche Sicht) und Methodenintegration (mit dem Ziel Validierung von Ergebnissen). Unabhängig davon, ob Methoden im Forschungsprozess integriert oder miteinander kombiniert werden, besteht das Ziel von Mixed-Methods-Studien immer darin, die unterschiedlichen Forschungsergebnisse anschließend in Beziehung zueinander zu setzen - hier wird entsprechend von der Integration von Ergebnissen gesprochen (Kelle und Buchholtz 2015). Dabei sind unter Integration je nach Forschungsdesign sowohl die Überführung (Transformation) und Einbettung von Forschungsergebnissen einer Perspektive in die andere zu verstehen (z. B. bei sequenziell angelegten Forschungsdesigns) als auch die Zusammenführung zweier oder mehrerer Perspektiven zur Schaffung einer neuen Perspektive (z.B. bei parallel angelegten Forschungsdesigns) (Creswell und Plano Clark 2018; Schoonenboom und Johnson 2017; Kuckarts 2014).

Zur begrifflichen Klärung von Mixed Methods kann aufbauend auf diesem Verständnis von Triangulation auf die Definition verwiesen werden, die Johnson et al. 
(2007) anhand einer Synthese von 19 Definitionen führender Mixed-Methods-Forschenden gegeben haben:

Mixed methods research is the type of research in which a researcher or team of researchers combines elements of qualitative and quantitative research approaches (e.g., use of qualitative and quantitative viewpoints, data collection, analysis, inference techniques) for the broad purposes of breadth and depth of understanding and corroboration. [...] A mixed methods study would involve mixing within a single study; a mixed method program would involve mixing within a program of research and the mixing might occur across a closely related set of studies. (S. 123)

Dabei suggeriert das Verwenden einer umfassenden Definition allerdings eine definitorische Einheit in Bezug auf Mixed Methods, die bislang innerhalb der MixedMethods-Community so nicht gegeben ist. Da die Diskussion um Mixed Methods in den Sozialwissenschaften beheimatet ist, spiegelt der Diskurs um Kombination bzw. Integration qualitativer und quantitativer Methoden implizit das sozialwissenschaftliche Methodenverständnis wider (Johnson et al. 2007; Johnson und Christensen 2017; Johnson und Onwuegbuzie 2004). Der Begriff „Mixed Methods“ wird daher von einigen Vertretern innerhalb der Sozialwissenschaften festgelegt auf die Kombination bzw. die Integration qualitativer und quantitativer Methoden, während für die Kombination bzw. die Integration anderer Forschungsmethoden (oder z. B. auch ausschließlich qualitativer Methoden) der weiter gefasste Begriff „Multi-Methods“ verwendet wird (Baur et al. 2017). Mittlerweile gibt es Vertreterinnen und Vertreter innerhalb der Mixed-Methods-Community, die die Festlegung auf qualitativ und quantitativ als überholt ansehen (z. B. Bazeley 2017). Sie bemängeln, dass Studien, die sich wissenschaftlicher Methoden bedienen, die sich nicht eindeutig qualitativer oder quantitativer Forschung zuordnen lassen, durch eine zu rigide Definition von Mixed Methods ausgeschlossen wären (Mertens et al. 2016; Morgan 2018). Eine enge Definition verdeckt aber auch die Kreativität und den Innovationsgehalt vieler Mixed-Methods-Studien, die teilweise unter nicht exakt klassifizierbaren Forschungsbedingungen entstehen. Die Mixed-Methods-Community formuliert daher den Minimalstandard von Mixed-Methods-Studien lediglich anhand zweier Kernkriterien: ,, use of more than one method, methodology, approach, theoretical or paradigmatic framework " and ,integration of results from those different components “" (Mertens et al. 2016, S. 4). Bei den Definitionen ist auffällig, dass sowohl Kombination als auch Integration nicht allein auf Daten oder Methoden festgelegt sind, sondern sich auch auf miteinander kombinierte Methodologien, Forschungsansätze, theoretische Frameworks und Techniken der Analyse erstrecken können, die in den Definitionen daher übergreifend ,elements“ oder „,components“ genannt werden (Schoonenboom und Johnson 2017). Die Definitionen lassen somit ein breites Verständnis des Integrationsbegriffs erkennen, bei dem sich Integration sogar auf 
die Ebene der den Methoden zu Grunde liegenden Forschungsparadigmen ${ }^{2}$ erstrecken kann (vgl. hierzu auch Greene 2015 und Johnson und Christensen 2017). Die Idee hinter diesem breiten Verständnis ist stets, dass die Stärken und Schwächen der jeweiligen Forschungsansätze bzw. Elemente vereint bzw. kompensiert werden können. Das gegenseitige Vorhalten von Schwächen qualitativer und quantitativer methodologischer Ansätze war noch bis in die 1980er Jahre Gegenstand der sogenannten „Paradigm Wars“ (Gage 1989) innerhalb der Sozialwissenschaften, und noch heute stehen Vertreter der sogenannten „Inkompatibilitätsthese“ zur Unvereinbarkeit von Forschungsparadigmen (Howe 1988) der gesamten MMF kritisch gegenüber.

Die Mixed-Methods-Bewegung hat sich jedoch seit den 1990er Jahren mehr und mehr zum Ziel gesetzt, Grabenkämpfe zwischen puristischen Vertretern qualitativer und quantitativer Paradigmen zu überwinden (Johnson und Onwuegbuzie 2004) und sich als verbindendes ,drittes Paradigma“ zwischen den etablierten Paradigmen zu positionieren. Dabei kann leicht übersehen werden, dass die Mixed-MethodsCommunity seit langem über eine wissenschaftsphilosophische Fundierung des Forschungsansatzes diskutiert, vor deren Hintergrund sich auch die Anknüpfungsmöglichkeiten für MMF in spezifischen Disziplinen genauer beschreiben lassen. Die Tragweite des breiten Verständnisses des Integrationsbegriffes zeigt sich, wenn Forschungsmethoden und -paradigmen der Mathematikdidaktik betrachtet werden (vgl. Abschn. 5).

\section{Wissenschaftsphilosophische Grundlagen der MMF}

\subsection{Paradigmengebundenheit von Forschungsmethoden}

Der Begriff Methode und auch der ihm verwandte Begriff Methodologie stammen etymologisch vom griechischen $\mu \dot{\varepsilon} \theta$ odo $\varsigma$ ab, was etwa „Weg zu etwas hin“, „Gang einer Untersuchung“ oder „Verfolgen von Wissen“ bedeutet (Beer 2008). Während der Terminus Methode sich begrifflich auf nach festen Regeln geordnete Verfahren zur Datenerhebung oder -analyse innerhalb wissenschaftlicher Studien bezieht, steht Methodologie eher für die Lehre von verschiedenen wissenschaftlichen Methoden als Teildisziplin der allgemeinen Wissenschaftstheorie oder auch für die Beschreibung der theoretischen Verankerung von Methoden innerhalb einzelner Wissenschaften (Beer 2008; Mittelstraß 2013). Konkretisiert in Bezug auf Forschung bezeichnet der Begriff Methodologie dementsprechend ein ,,voraussetzungsvolles Forschungsvorgehen hinsichtlich der philosophischen Annahmen, der Interpretationen und dem Verfassen der Ergebnisse“" (Kuckarts 2014, S. 22). Methodologie bezieht damit die theoretische(n) Position(en) Forschender bei der Auswahl und bei der Anwendung von Methoden innerhalb einer Studie sowie bei der Abgrenzung zu anderen theo-

\footnotetext{
2 Der Paradigmenbegriff meint in diesem Fall den konsensuellen Bestand von erkenntnistheoretischen Grundhaltungen, Überzeugungen und Praktiken von Forschenden einer entsprechenden Wissenschaft, die beeinflussen, wie sowohl die Forschungsfragen, die untersucht werden, als auch die Methoden, mit denen sie untersucht werden, ausgewählt werden (Morgan 2007).
} 
retischen Positionen (und anderen wissenschaftlichen Disziplinen) ein (Mittelstraß 2013). Unter anderem beschreibt etwa Salzman (2006), dass es somit auch verschiedene Methodologien geben kann, beispielsweise interpretative oder analytische, aber auch feministische oder marxistische (S. 366). Es erscheint daher sinnvoll, im Zusammenhang mit Mixed Methods von einzelnen qualitativen oder quantitativen sozialwissenschaftlichen oder eben auch spezifisch mathematikdidaktischen Forschungsmethoden zu sprechen, von Methodologie aber nur in Bezug auf eine den einzelnen Methoden übergeordnete Ebene, bei der theoretische Gesichtspunkte und die wissenschaftstheoretische Verankerung entsprechend reflektiert werden (wobei es hierbei nicht ausgeschlossen ist, verschiedenartige Methodologien zu unterscheiden). Folgt man der Prämisse einer paradigmengebundenen Methodologie, ergibt sich eine Methodologie nicht zuletzt aus den Charakteristika eines zu Grunde liegenden Forschungsparadigmas, wie beispielsweise dem Konstruktivismus oder dem Realismus. In den 1970er, 1980er und 1990er Jahren war es unter anderem Egon Guba, der einen Dialog zwischen unterschiedlichen Paradigmen zu etablieren versuchte. Er wies darauf hin, dass Paradigmen durch verschiedene Dimensionen charakterisiert seien. Zum einen spielt ihre Ontologie eine Rolle, also die Frage, wie Realität innerhalb eines Paradigmas konzeptualisiert ist. Des Weiteren ergibt sich eine Charakterisierung auch durch ihre Epistemologie, also die Frage, welche Voraussetzungen für Erkenntnis und für das Zustandekommen von Wissen gegeben sein müssen und was akzeptierbares Wissen ausmacht. Schließlich sind Paradigmen auch durch ihre Methodologie charakterisiert, also die Frage, wie innerhalb des Paradigmas Wissen systematisch erschlossen werden kann (Guba 1990). Guba, ein führender qualitativer Purist, zielte dabei allerdings nicht auf eine wie auch immer geartete Vereinbarkeit unterschiedlicher Paradigmen ab. Er äußert sich im Gegenteil im Sinne der Inkompatibilitätsthese: ,accommodation between paradigms is impossible [...] we are led to vastly diverse, disparate, and totally antithetical ends" (Guba 1990). Vertreter der Inkompatibilitätsthese argumentieren vor allem für eine starke Abhängigkeit zwischen Paradigma und Methodologie und sehen unüberbrückbare Gegensätze zwischen den wissenschaftstheoretischen Grundpositionen unterschiedlicher Paradigmen. Sie lehnen daher jegliche Vereinigung über ein rein oberflächliches Niveau oder über mehr als nur innerhalb eines Paradigmas hinaus ab. Die Idee, dass einzelne Forschungsmethoden eben jenen epistemologischen und ontologischen Verpflichtungen anhängig sind, wird dementsprechend als eines der Hauptargumente gegen den Mixed-Methods-Ansatz hervorgebracht. Die von ihnen betonte starke Bindung von Methode, Methodologie und Paradigma ist allerdings immer wieder kritisiert worden (Hammersley 2002):

[The] paradigm view of the relationship between qualitative and quantitative approaches is empirically inaccurate [...] it implies that we are faced with two homogenous traditions that are internally coherent and based upon opposed philosophical views. In fact [...] there is no fixed relationship between particular philosophical views and the use of particular methods. Furthermore, even the most superficial peruse of philosophical literature shows that there are not 
simply two epistemological positions from which to choose. [...] In epistemolo$\mathrm{gy}$, as in methodology, such dichotomies obscure the range of possibilities that is open to us. (S. 167)

Eine derartig strenge Abhängigkeit kann insbesondere für sozialwissenschaftliche Forschung nicht ausgemacht werden, und einzelne Methoden können somit durchaus in den Dienst unterschiedlicher Paradigmen gestellt werden (Bryman 2016). Die meisten Mixed-Methods-Forschenden weisen die starke Verankerung von Methoden innerhalb von miteinander unvereinbaren Paradigmen daher zurück und nehmen entweder eine aparadigmatische Position ein oder fordern ein eigenes erkenntnistheoretisches Paradigma für die MMF (Kelle 2017).

\subsection{Paradigmen der MMF}

Eine aparadigmatische Position zu Mixed Methods reduziert den Gebrauch wissenschaftlicher Forschungsmethoden auf ein eher technisch-handwerkliches Vorgehen, das überwiegend unabhängig von philosophischen und epistemologischen Debatten ist und eher von praktischer Erfahrung und „Commonsense“ geprägt ist (Hammersley 2002). Hier liegen jedoch die Gefahren der eingangs geschilderten Qualitätsprobleme von Mixed-Methods-Studien. Die andere Position verortet die Mixed-Methods-Bewegung innerhalb der philosophischen Schule des US-amerikanischen Pragmatismus, der auf den Arbeiten von Peirce, James und John Dewey beruht (Johnson und Onwuegbuzie 2004). Der Pragmatismus wird dabei als begleitende Philosophie herangezogen, und zahlreiche namhafte Autorinnen und Autoren der Mixed-Methods-Community beschreiben ihn als das der MMF zu Grunde liegende Paradigma (Tashakkori und Teddlie 2003; Johnson und Onwuegbuzie 2004; Howe 1988). Die pragmatische Sicht zeichnet sich durch den Grundsatz aus, dass sie den (provisorischen) Wahrheitsgehalt wissenschaftlicher Aussagen an empirischen Erfahrungen oder an praktischen Konsequenzen dieser Aussagen in der Lebenswelt misst. Damit wird ein bedarfs- oder kontingenzbasierter Ansatz bei der Auswahl von Forschungsmethoden impliziert, bei dem der praktischen Verwertbarkeit wissenschaftlicher Befunde im Sinne eines ,what works“ ein höherer Stellenwert beigemessen wird als einer ontologischen oder epistemologischen Paradigmengebundenheit (Johnson und Onwuegbuzie 2004). Konkret bedeutet das in Bezug auf die MMF, dass jeweils diejenigen Forschungsmethoden ausgewählt werden, die die Beantwortung der Forschungsfrage am besten gewährleisten. Außerdem werden hierbei unterschiedliche ontologische Positionen ausgeblendet, solange sie keinen Unterschied in Hinblick auf die praktischen Konsequenzen der Durchführung einer Studie ausmachen. Vor diesem Hintergrund lässt sich das breite Integrationsverständnis der Definitionen von Mixed Methods besser verstehen: Nicht die paradigmatische Gebundenheit einer Methode oder einer Methodologie sind entscheidend für die Verknüpfung oder eben die Inkommensurabilität unterschiedlicher Elemente im Forschungsprozess, sondern der Nutzen und die Konsequenzen, den eine pragmatische Verschränkung dieser Elemente mit sich bringt. Während das übergeordnete Ziel eines MixedMethods-Ansatzes dabei immer ist, die Schlussfolgerungen einer Studie zu vertiefen und zu stärken, können basierend auf diesem Verständnis bestimmte allgemein- 
methodische Ziele herausgearbeitet werden, die bei einer Kombination bzw. Integration unterschiedlicher Forschungsmethoden verfolgt werden (siehe Bryman 2016). Diese werden daher in vielen Mixed-Methods-Studien als Begründung für das Vorgehen herangezogen. $\mathrm{Zu}$ den bekanntesten zählt die Klassifikation von Greene et al. (1989), die auf einer Analyse zahlreicher Mixed-Methods-Studien beruht. Identifiziert werden fünf teleologische Ausrichtungen für die Kombination bzw. Integration von Forschungsmethoden (vgl. S. 259):

1. Triangulation, d.h. die Suche nach Konvergenz, Bestätigung und Übereinstimmung der Ergebnisse aus verschiedenen Methoden, also die gegenseitige Validierung von Forschungsergebnissen.

2. Komplementarität, d.h. die Suche nach Vertiefung, Verbesserung, Veranschaulichung oder Klärung der Ergebnisse einer Methode mit den Ergebnissen der anderen Methode. Ziel des Kombinierens und Integrierens ist es hierbei, den Forschungsgegenstand ganzheitlich zu verstehen.

3. Entwicklung, d.h. das Bestreben, die Ergebnisse einer Methode zu verwenden, um die Entwicklung einer anderen Methode zu unterstützen.

4. Initiierung, d.h. die Suche nach paradoxen und widersprüchlichen Erkenntnissen, nach neuen Perspektiven von Rahmenmodellen. Es kann dabei auch um die Neufassung von Ergebnissen einer Methode mit Fragen oder Ergebnissen einer anderen Methode gehen.

5. Erweiterung, d.h. der Versuch, Breite und Reichweite der Untersuchung zu erweitern, indem verschiedene Methoden für unterschiedliche Untersuchungskomponenten verwendet werden.

Diese pragmatische Position ist mit Blick auf Voraussetzungen und Qualitätskriterien aus zwei Gründen problematisch: Einerseits liegt hier ein gewisser methodischer Relativismus vor, andererseits erfordert das pragmatische Wahrheitskriterium, dass die Bewertung von praktischen Konsequenzen in der Methodenkombination oder -integration stets möglich ist und nicht intersubjektiv variiert (vgl. Kelle 2017). Auch vor dem Hintergrund der eingangs geäußerten Kritik an fehlenden Begründungen für die Wahl von Mixed-Methods-Forschungsdesigns erscheint eine rein pragmatische Orientierung mathematikdidaktischer Mixed-Methods-Studien daher fragwürdig. Zu sehr dominieren in diesem Fall technische Beschreibungen und die Orientierung an bestimmten Forschungsdesigns das methodische Vorgehen, wodurch nicht deutlich genug wird, welchen disziplinspezifischen Mehrwert etwa eine Methodenkombination oder -integration mit sich bringen kann. Eine andere Perspektive ergibt sich allerdings, wenn der Blick stärker auf den Gegenstandsbereich der Forschung gelenkt wird.

Es ist nicht nötig, die Annahme der starken Verbindung zwischen Methodologie und Paradigma gänzlich zu verwerfen (aparadigmatische Position) oder auszublenden (pragmatische Position), um einen methodischen Pluralismus zu befürworten. Wichtig ist dabei, ontologische und epistemologische Aspekte im Gebrauch von Methoden bei den Überlegungen zu berücksichtigen, denn die Formulierung einer alles bestimmenden Forschungsfrage erfolgt zumindest aus einer impliziten ontologischen Position heraus. Danermark et al. (2019) wenden daher ein, dass es Forschenden nicht möglich ist, der ontologischen Dimension ihres Erkenntnisinteresses zu entrin- 
nen. Innerhalb der Mixed-Methods-Community wird diese Position dem Realismus als bestimmendem Paradigma zugeordnet (Maxwell und Mittapalli 2010). Zur Beschreibung der Position werden allerdings verschiedene Begriffe verwendet (Lakoff 1987), einschließlich des auf Bhaskar oder Archer zurückgehenden Critical Realism (Bhaskar 1978, 1989; Archer et al. 1998), dem hier eine leitende Rolle zukommt.

Die ontologischen Grundlagen des Critical Realism beziehen sich auf die Annahme der Existenz einer Wirklichkeit unabhängig von Wissen über die Wirklichkeit, wobei zwischen einer der Beobachtung nicht zugänglichen ,Realität“ und der ,,beobachtbaren Welt" unterschieden wird. Lakoff (1987) definiert diese Unterscheidung zwischen objektivistischen und realistischen Ansichten wie folgt:

Scientific objectivism claims that there is only one fully correct way in which reality can be divided up into objects, properties, and relations. [...] Scientific realism, on the other hand, assumes that ,the world is the way it is“, while acknowledging that there can be more than one scientifically correct way of understanding reality in terms of conceptual schemes with different objects and categories of objects. (S. 265)

Die Welt wird demnach durch das konstruiert, was „,beobachtbar“ ist. Dem Critical Realism zufolge verursachen dabei nichtbeobachtbare Strukturen (unobservable structures) beobachtbare Ereignisse, und die Welt kann nur verstanden werden, wenn die zu Grunde liegenden Mechanismen (underlying mechanisms) verstanden werden, von denen die Ereignisse erzeugt werden (Danermark et al. 2019). Das bedeutet, dass untersucht wird, in welchen relationalen Verhältnissen Strukturen zu anderen Strukturen stehen. Da das Wissen über diese Strukturen aber immer nur kontextuell, perspektivisch und damit fehlbar sein kann, hält der Critical Realism in dieser Position an einem ontologischen Realismus fest, während er eine Form von epistemologischem Relativismus oder Konstruktivismus akzeptiert (Archer et al. 1998). ${ }^{3}$ Die Realität wird hier als strukturiert und geschichtet (stratified) konzipiert, womit gleichzeitig die Forderung an Forschende verknüpft ist, auf verschiedenen Analyseebenen geeignete - also an unterschiedliche Schichten des Forschungsgegenstandes angepasste - Forschungsmethoden zur Untersuchung einzusetzen, um jede Schicht des Untersuchungsobjekts zu verstehen.

Vertreter des Critical Realism kritisieren den pragmatischen Ansatz der MMF für die Missachtung dieser Voraussetzungen: ,the practical and the empirical take precedence over the ontological and the epistemological“ (Danermark et al. 2019, S. 167). Die theoretisch auf dem Critical Realism aufbauende MMF befasst sich demnach mehr mit der Konzeptualisierung der Realität in verschiedenen Schichten. Epistemologisch steht dabei im Vordergrund, wie Wissen über Forschungsgegenstände auf verschiedenen Analyseebenen sowohl durch quantitative als auch durch qualitative Methoden erlangt werden kann (Riazi und Candlin 2014). Die Implikation einer solchen kritischen realistischen Perspektive ist, dass es möglich ist, im Einklang mit ontologischen Überlegungen zum Untersuchungsgegenstand von verschiedenen Forschungsmethoden oder -paradigmen in systematischer Weise Gebrauch zu ma-

\footnotetext{
${ }^{3}$ Damit unterscheidet sich der Critical Realism auch von der Position des kritischen Rationalismus nach Popper.
} 
chen, so dass unterschiedliche Schichten des Gegenstandes durch jeweils geeignete Methoden untersucht werden können und auch die Inkompatibilitätsthese zurückgewiesen werden kann (Riazi und Candlin 2014; Riazi 2016). Mixed-Methods-Studien folgen damit weniger rein pragmatischen Entscheidungen, die zu parallelen oder, wie Riazi und Candlin (2014) bemerken, ,eklektischen“ oberflächlichen Kombinationen verschiedener Methoden führen können.

Im Folgenden (Abschn. 4 und 5) werden anhand von diesem MMF-Verständnis die disziplinspezifischen Annahmen für Mixed-Methods-Studien in der Mathematikdidaktik spezifiziert. Dabei spielt einerseits die Stratifizierung mathematikdidaktischer Forschungsgegenstände eine zentrale Rolle. Diese befinden sich im Spannungsfeld verschiedener Disziplinen wie Mathematik, Psychologie, Soziologie, Philosophie oder Erziehungswissenschaft, fokussieren auf verschiedene Akteure auf unterschiedlichen bildungsrelevanten Ebenen und stehen zusätzlich in einem systemischen Verhältnis zwischen Theorie und Praxis. Andererseits geraten aber auch unterschiedliche epistemologische Ausrichtungen innerhalb der mathematikdidaktischen Forschung in den Blick, die jeweils von unterschiedlichen Forschungsmethoden Gebrauch machen, um spezifische Aspekte von Forschungsgegenständen zu untersuchen.

\section{Ontologie der Forschungsgegenstände in der Mathematikdidaktik}

Trotz jahrzehntelanger Diskussionen über den Gegenstandsbereich mathematikdidaktischer Forschung (exemplarisch Bigalke 1974; Wittmann 1992; Schoenfeld 2002, 2016), ist nicht abzusehen, dass sich ein diesbezüglicher Konsens bildet (vgl. Sierpinska und Kilpatrick 1998). Einigkeit scheint aber zumindest darüber zu bestehen, dass das mathematische Wissen in Bildungsprozessen einen zentralen Stellenwert als Forschungsobjekt einnimmt. Forschungsgegenstände in der Mathematikdidaktik beziehen sich aus ontologischer Sicht jedoch weniger auf das eigentliche mathematische Wissen, also auf das streng logische und hierarchisch strukturierte systematische Gefüge aus abstrakten und allgemeingültigen Aussagen über Strukturen, das mit Hilfe der axiomatischen Methode beschrieben wird. Vielmehr muss das mathematische Wissen im Rahmen mathematikdidaktischer Forschung relational „im Kontext sozialer Prozesse der Vermittlung“ verortet werden (Steinbring 1998, S. 161), da ,mathematische Begriffe, wie Zahlen, Funktionen, Vektoren - die ja keine Dinge im herkömmlichen Sinne sind, sondern Beziehungen verkörpern - in der Wahrnehmung oder auch in einer anderen intuitiven Erfahrung nicht direkt zugänglich sind - wie zum Beispiel reale oder physische Gegenstände -, sondern durch Zeichen bzw. Symbole repräsentiert werden müssen“ (Steinbring 1998, S. 162). Hierin lässt sich ein Anknüpfungspunkt zum Critical Realism ausmachen. Das abstrakte (theoretische) mathematische Wissen wird in Bildungskontexten als ein nicht direkt der Beobachtung zugängliches (aber strukturiertes) Geflecht von Beziehungen verstanden, das sich jedoch durch den personengebundenen Gebrauch und durch die Konstruktion eines intersubjektiv nachvollziehbaren semiotischen Zeichensystems (Steinbring 1998) als einer empirischen Untersuchung zugänglich erweist. Damit steht weniger der Aufbau und die Verifikation theoretischen mathematischen Wis- 
sens im Mittelpunkt mathematikdidaktischer Forschung (worin eine fundamentale Abgrenzung zur Mathematik besteht), sondern die Erklärung der Mechanismen und Voraussetzungen des Vermittlungs-, Aneignungs- und Entwicklungsprozesses (Sierpinska und Lerman 1996). Diese Prozesse können in schulische, kulturelle und gesellschaftliche Zusammenhänge eingebettet sein. Zusätzlich werden die Prozesse auch unter einer normativen Perspektive der „Optimierung“ betrachtet (Otte 1982; zitiert in Steiner 1985; Bigalke 1974).

Als illustrierende Beispiele lassen sich - ohne den Anspruch auf Vollständigkeit - einige genuin mathematikdidaktische Forschungsgegenstände identifizieren, die von einer Vielzahl Forscherinnen und Forscher im Rahmen von Studien untersucht werden (für einen detaillierten Überblick vgl. Griesel 1974; Ernest 1998; Schoenfeld 2016; Steinbring 1998; Wittmann 1992):

- die Natur von Mathematik und mathematischem Schulwissen, einschließlich epistemologischer, historischer und theoretischer Fragen,

- Anknüpfungspunkte für die Mathematik in Realität und Freizeit,

- mathematische Arbeitsweisen, Prozesse und Kompetenzen (z. B. Beweisen, Modellieren, Problemlösen),

- das individuelle Lernen von Mathematik, einschließlich kognitiver und affektiver psychologischer Aspekte und ihrer jeweiligen Voraussetzungen (z. B. Repräsentationen, Überzeugungen, Motivation, Heuristiken),

- Tätigkeiten des Lehrens von Mathematik (z.B. inhaltliche Strukturierung, Fördern, Erklären, Leistungsmessung),

- Entwicklung von Unterrichtseinheiten und auf Lehrplänen basierenden Materialien, für alle Arten von mathematischen Inhalten sowie ihre Bewertung im Hinblick auf Durchführbarkeit und Wirksamkeit,

- die Ziele und der Bildungsgehalt des Mathematikunterrichts und der Schulbildung sowie der Aufbau von Curricula,

- der Mathematikunterricht und verschiedene Unterrichtsformen und -methoden (z.B. Unterrichtsqualität, kooperatives Lernen, entdeckendes Lernen),

- Lehrbücher, Lehrpläne, Lehrmaterialien und digitale Werkzeuge, die im Zusammenhang mit dem Lehren und Lernen im Mathematikunterricht eingesetzt werden,

- soziale Kontexte beim Lernen und Lehren von Mathematik (z. B. Heterogenität, Sprache, Kultur, Geschlecht),

- Interaktionen und Beziehungen zwischen Akteuren und in Bezug auf mathematische Inhalte (z. B. Schüler-Lehrer-Interaktionen),

- die Lehrerausbildung im Fach Mathematik und ihre Institutionen und Systeme (z.B. Professionsforschung, vergleichende Studien) sowie

- die Natur der mathematischen Bildung, ihre Erkenntnistheorie und ihre Methodik.

Die im Critical Realism angenommene Mehrschichtigkeit der Forschungsgegenstände lässt sich auf die mathematikdidaktische Forschung übertragen. Deren Forschungsgegenstände zeichnen sich insgesamt durch eine hohe Komplexität aus, die Forschungszugänge auf unterschiedlichen Analyseebenen bereithält. Diese Komplexität ist u. a. durch die besondere Stellung der abstrakten Mathematik als Bezugswissenschaft gegeben, die Forschungsgegenstände auf einer theoretischen Ebene formal strukturiert. Die von dort bezogenen Forschungsgegenstände müssen unter 
einer interdisziplinären und integrierenden Perspektive in „Korrespondenz [...] mit adäquaten erziehungswissenschaftlichen, psychologischen, soziologischen und philosophischen Erkenntnissen, Fragestellungen und Arbeitsweisen“ (Bigalke 1974) gebracht werden (Wittmann 1992; Bartolini-Bussi und Bazzini 2003) und sind dementsprechend stratifiziert. Weiterhin sind die untersuchten Gegenstände aber auch durch ein komplexes systemisches Gefüge aus unterschiedlichen intra- bzw. interpersonalen Zusammenhängen und aus interagierenden Instanzen geprägt (Steiner 1985), was eine entsprechende Analyseebene in Bezug auf soziale und psychische Schichten der Forschungsgegenstände eröffnet. Beispiele für interpersonelle Strukturen, die sich in der mathematikdidaktischen Forschung wiederfinden, sind u.a. das Schüler-Lehrer Verhältnis, Gesprächs- und Interaktionsmuster oder Gruppendynamiken. Intrapersonelle Strukturen werden beispielsweise bei der Untersuchung von Motivation, Emotionen sowie bei der kognitiven Verarbeitung von mathematischen Inhalten, Handlungsprozessen, Wissen oder Überzeugungen berücksichtigt. Über diese Strukturen hinaus können sich mathematikdidaktische Forschungsgegenstände über verschiedene interagierende Instanzen auf unterschiedlichen Ebenen erstrecken. So kann etwa die Mikroebene (Individuum), die Mesoebene (z. B. Gruppe, Klasse, Schule) und/oder die Makroebene (z. B. Länderebene oder Bildungssystem) fokussiert werden. Nicht zuletzt sind mathematikdidaktische Forschungsgegenstände auch in Bezug auf eine zeitliche und kulturelle Analyseebene eingebunden innerhalb von Handlungsdynamiken, aber auch in bildungspolitische und gesellschaftliche Veränderungsprozesse (Schoenfeld 2016; Steinbring 1998). Einerseits unterliegt beispielsweise der Mathematikunterricht oder die Mathematiklehrerausbildung langfristigen gesellschaftlichen und dynamischen Veränderungsprozessen, wie sie typisch in sozialen Bildungsstrukturen sind (Schoenfeld 2016). Forschungsarbeiten zur Geschichte der Mathematik und der Mathematikdidaktik versuchen, diese Entwicklungen nachzuzeichnen. Andererseits können untersuchte Forschungsgegenstände selbst zeitliche Prozesse sein. Hierunter fallen etwa zeitliche Verläufe von Lernprozessen aller Art oder auch die Entwicklung von Bildungssystemen, wie dies beispielsweise in Panelstudien oder Quasi-Längsschnitten im Rahmen von (internationalen) Vergleichsstudien geschieht. Zusätzlich trägt zur Mehrschichtigkeit auch bei, dass die Mathematikdidaktik in ein systemisches und durch diverse Akteure geprägtes Gefüge aus Theorie und Praxis eingebettet ist (u.a. Steinbring 1998). Konsensfähig erscheint innerhalb der Mathematikdidaktik daher die Auffassung über die untrennbare Verbindung von Wissenschaft und Ausbildungspraxis (z.B. Burkhardt und Schoenfeld 2003; Bartolini-Bussi und Bazzini 2003), so dass mathematikdidaktische Forschungsgegenstände auch in Hinblick auf eine praktische Analyseebene strukturiert sein können. So kann von einer Wechselbeziehung zwischen Praxis und wissenschaftlicher Theorie ausgegangen werden (vgl. Steinbring 1998), die sich einerseits in einer konstruktiven, auf didaktische Entwicklungsarbeiten fokussierten Forschungsdimension zeigt. Als fachdidaktische Entwicklungen können beispielsweise Analysen zur Natur des mathematischen Wissens, aber auch alle Arten von stoffdidaktisch entwickelten Lehrmaterialien, digitalen Werkzeugen, Curricula oder Lehrstrukturen verstanden werden, die im Rahmen von Interventionen oder Implementationen eingesetzt werden. Andererseits wirkt die Praxis im Rahmen einer analytischen Forschungsdimension auf die theoretische Konstruktion 
von Lernumgebungen zurück. In einer entsprechenden analytischen Forschungsdimension finden sich alle Arten von empirischen Untersuchungen wieder, die individuelle und unterrichtliche Lernprozesse und soziale Interaktionen beispielsweise $\mathrm{zu}$ verschiedenen mathematischen Bereichen untersuchen, wie etwa dem geometrischen und räumlichen Denken, dem stochastischen Denken oder dem algebraischen Denken.

\section{Epistemologien und Methodenpluralismus in der Mathematikdidaktik}

\subsection{Epistemologische Ausrichtungen mathematikdidaktischer Forschung}

Die Bestimmung eines mathematikdidaktischen Forschungsgegenstandes und die Auswahl von Forschungsmethoden, die entsprechende gegenstandsbezogene Forschungsfragen beantworten können, erfolgt immer vor dem Hintergrund epistemologischer Überzeugungen zum Wesen mathematikdidaktischer Forschung bzw. unterschiedlicher Forschungsparadigmen (für einen Überblick vgl. etwa Sierpinska und Lerman 1996; Ernest 2018). So unterscheidet Bishop (1992) unter Rückbezug auf den erziehungswissenschaftlichen Paradigmenbegriff von Habermas (1972) drei unterschiedliche Traditionen mathematikdidaktischer Forschung: ${ }^{4}$ (1) Eine pädagogische oder erziehungswissenschaftliche Tradition, deren Ziel es ist, direkte Verbesserungen der Praxis zu bewirken und die sich im Wesentlichen auf das Ausprobieren und das Beobachten konzentriert. Dabei verweist Bishop etwa auf frühe Vertreter wie Polya oder Freudenthal. Auch eine Vielzahl mathematikdidaktischer Studien aus den Vereinigten Staaten orientiert sich an dieser Forschungstradition (vgl. Schoenfeld 2016). Methodisch ist sie im Bereich der Beobachtung und der Verbesserung von Lehrtätigkeiten verortet, wobei u. a. exemplarisch und auch fallbasiert von Expertenlehrkräften gewonnene Erkenntnisse verallgemeinert werden. Bishop unterscheidet weiter (2) die Tradition des ,empirical scientist“ (Bishop 1992; S. 712), deren Ziel es ist, auf Grundlage von empirischen Daten die Praxis zu analysieren und Erklärungsmodelle zu generieren. Gerade in dieser Tradition sieht Bishop eine starke methodologische Fixierung, die mathematikdidaktische Forschung als Experimentalwissenschaft versteht und Theorie zur Erklärung von wissenschaftlichen Evidenzen heranzieht. Dabei stehen sich qualitative und quantitative Forschungsmethoden gegenüber. Ernest $(1998,2018)$ unterscheidet daher innerhalb dieser Tradition (ebenfalls unter Rückbezug auf Habermas) noch einmal zwischen dem ,scientific research paradigm “ (Ernest 1998, S. 77), dessen Ursprung er im kritischen Rationalismus sieht, und das quantitative sozialwissenschaftliche Forschungsmethoden in der mathematikdidaktischen Forschung priorisiert (vgl. „(Post-)Positivist Paradigm“ bei Scheiner 2019), und dem ,interpretative research paradigm“ (S. 77), das sich auf eher qualitative Methoden der Anthropologie und der Ethnographie beruft und

\footnotetext{
${ }^{4}$ Um hier präzise zu bleiben, ergänzt Bishop (1992, S. 712) jedoch: „A research tradition is very different from a research paradigm. One doesn't choose a research tradition as one might choose a research paradigm; one is part of it, as a result of upbringing, education, cultural background, and research training.".
} 
oft die epistemologische Grundlage für konstruktivistisch angelegte Forschungsansätze bildet (vgl. Sierpinska und Lerman 1996; Scheiner 2019). Zusätzlich ergänzt Ernest (1998) hier um ein kritisch-theoretisches Paradigma, dessen Gegenstand soziale Transformationsprozesse sind und auf das auch Scheiner (2019) unter dem Stichwort „Transformative Paradigm“ (S. 519) in einer neueren Arbeit zu mathematikdidaktischen Paradigmen hinweist. Als dritte Forschungstradition (3) führt Bishop die ,scholastic-philosopher tradition“ an (Bishop 1992), die die mathematikdidaktische Forschung als einen rigoros argumentierten theoretischen Reflexionsprozess begreift. Das epistemologische Ziel aus dieser Perspektive ist es, eine argumentierte Position $\mathrm{zu}$ theoriegeleiteten (auch didaktisch motivierten mathematischen) Forschungsfragen und zur Entwicklung von Lehrplänen aufzubauen - stark basierend auf mathematischen Einsichten und logischer Strenge. Die Erfassung und Herausarbeitung des mathematischen Kerns eines Unterrichtsinhalts im Rahmen der „didaktisch orientierten Sachanalyse“ (Griesel 1972, 1974, 1997) in klassisch stoffdidaktischen Arbeiten (z. B. Kirsch 1969) etwa fällt in diesen Bereich, oder auch die epistemologische Analyse mathematischer Konzepte (Brousseau 1981).

\subsection{Methodenpluralismus und Stoffdidaktik}

Entsprechend dieser Variation der epistemologischen Sichtweisen lässt sich innerhalb der Disziplin ein breites Spektrum mathematikdidaktischer Forschungsmethoden identifizieren (Schoenfeld 2002). Über methodische Ansätze ist innerhalb der mathematikdidaktischen Forschung im Rahmen von Aktualisierungen des fachlichen Selbstverständnisses daher immer wieder gestritten worden (z. B. Bigalke 1974; Sierpinska und Kilpatrick 1998). Dabei werden teilweise entgegengesetzte Positionen eingenommen. Grundsätzlich wird die Diversität von Forschungsmethoden, Methodologien und Paradigmen aber als Stärke der mathematikdidaktischen Forschung wahrgenommen (Ernest 2018). So können innerhalb der pädagogischen Tradition u. a. partizipative Forschungsmethoden der Aktionsforschung verwendet werden. Hierzu ist allerdings immer wieder auf die Gefahr einer reduktionistischen, weil unzureichend theoriebasierten Forschungsmethodik hingewiesen worden (Steiner 1985; Schoenfeld 2016; Winter 1985, Stichwort: Praktikerwissen). Die Tradition des empirical scientist übernimmt hingegen etablierte Forschungsmethoden aus anderen Bereichen, insbesondere aus den Nachbardisziplinen Sozialwissenschaft, Psychologie und Bildungsforschung und passt sie an die entsprechenden mathematikdidaktischen Fragestellungen an. Insbesondere Forschungsmethoden, die sich der qualitativ-interpretativen Methodologie zuordnen lassen, haben sich hierbei im Lauf der Zeit in der mathematikdidaktischen Forschung mehr und mehr etabliert (z. B. Bikner-Ahsbahs et al. 2015). Die epistemologisch eher scholastisch-philosophisch geprägte Position sieht hingegen die genuine mathematikdidaktische Forschungsmethode in der fachlichen Begründung von mathematikspezifischen Bildungszielen und der entsprechenden Auswahl, Strukturierung und fachdidaktischen Aufbereitung schulrelevanter Lerninhalte von der Fachmathematik aus - entsprechend etwa Griesels (1972) didaktisch orientierter mathematischer Analyse. Dieser, insbesondere im deutschsprachigen Raum verbreitete und als „stoffdidaktische Methode“ oder „Stoffdidaktik“ bezeichnete Ansatz (für einen Überblick vgl. etwa Vohns 2007; 
Steinbring 2011) besteht darin, relevante mathematische Inhalte und Strukturen als Kern des Unterrichtsgegenstandes zu erfassen und in Form einer „Hintergrundtheorie“ zu formulieren, aber auch darin, sie für den geistigen Entwicklungsstand des Lernenden zugänglich zu machen und mit Blick auf die Anforderungen des Lehrens und Lernens anzupassen, ohne dabei mathematische Standards zu verfälschen (vgl. etwa Kirsch 1977). Steinbring (2011, S. 46) bezeichnet die Stoffdidaktik sogar als ein eigenes Forschungsparadigma, in dem ,the scientific elaboration of mathematical knowledge is the central and crucial means practiced for steering and optimizing mathematical instruction, learning and understanding processes." Dabei werden (zumindest ursprünglich) mathematische Arbeitsweisen und Methoden angewendet (Griesel 1974):

Die Forschungsmethoden dieses Bereiches sind mit denen der Mathematik identisch, so daß gelegentlich bei Außenstehenden der Eindruck entstanden ist, daß hier Mathematik (speziell Elementarmathematik) und nicht Didaktik der Mathematik betrieben werde. (S. 118)

Griesel (1972) räumt aber auch ein, dass eine alleinige mathematische Bearbeitung stoffdidaktischer Fragen ohne empirische Ergänzung für den mathematischen Lernprozess bedeutungslos sein mag. Dies kommt auch in der expliziten ,didaktischen Orientiertheit“" (S. 79) der stoffdidaktischen Methoden zum Ausdruck, die stets zum Ziel haben, den mathematischen Lernprozess zu organisieren, den Kern traditioneller Unterrichtspraxis herauszuarbeiten und individuelle Verstehenszugänge zu berücksichtigen (Griesel 1972; dazu auch Sträßer 2015). Weil sie sich in dieser Ausrichtung an ontologischen Gesichtspunkten des mathematischen Wissens in Bildungszusammenhängen orientieren, nehmen stoffdidaktische Methoden in der mathematikdidaktischen Forschung eine zentrale Rolle ein. Aktuelle Ausprägungen stoffdidaktischer Methoden greifen einzelne mathematische Themen oder auch ganze Stoffgebiete auf, die im Rahmen von sogenannten ,didaktischen Rekonstruktionen“ (Biehler und Blum 2016) für Lernende elementarisiert, d. h. zugänglich gemacht werden (Kirsch 1977). Dies vollzieht sich in einer Aufbereitung des Stoffes, die unterschiedliche lernpsychologische und mathematische Teilprozesse berücksichtigt, z.B. eine historische oder psychologische Genetisierung, eine Klassifizierung in Bezug auf Darstellungsebenen und epistemologische Zugänge, oder eine Analyse von Grundvorstellungen und -begriffen (Lambert 2014; Hoffkamp 2014).

Eine sowohl die empirische als auch die stoffdidaktische Perspektive integrierende Position im Hinblick auf die methodische Orientierung, die insbesondere das Verhältnis zwischen Theorie und Praxis innerhalb der mathematikdidaktischen Forschung aufgreift, nimmt die fachdidaktische Entwicklungsforschung ein, die ihren Ursprung im erziehungswissenschaftlichen Ansatz developmental or design research hat (Cobb et al. 2003; Van den Akker et al. 2006). Dabei wird der Forschungsvorgang als zyklischer Prozess gestaltet, der auf der fachlichen Spezifizierung und Strukturierung von Lerninhalten, auf der darauf aufbauenden Designentwicklung (Lernumgebungen) und auf der anschließenden empirisch beforschten Durchführung von Designexperimenten beruht (Hußmann und Prediger 2016; Prediger et al. 2012; Bakker und Van Eerde 2015). 


\section{Qualitätskriterien von Mixed-Methods-Studien in der mathematikdidaktischen Forschung}

Die methodologischen Voraussetzungen zur MMF einerseits und die Annahmen zu Forschungsgegenständen und unterschiedlichen epistemologischen Ausrichtungen mathematikdidaktischer Forschung andererseits können einen ersten Ansatzpunkt zur Formulierung von Qualitätskriterien für mathematikdidaktische Mixed-MethodsStudien bereitstellen.

Ein zentrales Kriterium für die Güte ist demnach, dass die Methodenkombination oder -integration nicht nur auf technischer Ebene entlang eines gewählten Forschungsdesigns realisiert wird, sondern dass die Studien darüber hinaus eine am Forschungsgegenstand orientierte Begründung des Forschungsansatzes beinhalten. Das bedeutet, dass über (pragmatische) allgemein-methodische Begründungen hinaus bei der Begründung dargelegt werden sollte, inwieweit der untersuchte Forschungsgegenstand eine ontologische Mehrschichtigkeit und Komplexität aufweist, die eine Kombination oder Integration von Forschungsmethoden überhaupt erst erforderlich macht. Insbesondere ist zu klären, inwiefern sich aus dieser Mehrschichtigkeit eine mathematikdidaktische Spezifität ergibt, das heißt, inwieweit sich innerhalb des Forschungsgegenstandes die Mathematik relational in gesellschaftlichen, kulturellen oder individuellen Zusammenhängen untersuchen lässt bzw. inwieweit sich mathematisches Wissen in Aneignungs- und Lehrprozessen als einer methodisch geleiteten Analyse zugänglich herausstellt ${ }^{5}$. Als hilfreich erweist sich hier, beim Formulieren von entsprechenden Forschungsfragen verschiedene Schichten des Gegenstands durch Teilfragen zu adressieren und die Teilfragen zu einer übergreifenden integrierten Forschungsfrage zu synthetisieren (s. Buchholtz 2019).

Entsprechend sollte als zweites Kriterium bei der Wahl der Methoden auch der epistemologische Standpunkt expliziert werden, und es sollte untersucht werden, inwieweit die zu Grunde liegenden (mathematikdidaktischen) Forschungsparadigmen unterschiedliche Schichten des Forschungsgegenstandes erschließen können. Ferner muss - um ein Mixed-Methods-Forschungsvorhaben von sogenannten MonoMethods-Studien abzugrenzen - deutlich werden, welcher Mehrwert für den Forschungsgegenstand sich aus einer Kombination bzw. Integration von Ergebnissen ergibt, die mit einzelnen Methoden allein nicht erfasst werden können.

Das bedeutet drittens, dass bei der Integration der Forschungsergebnisse durch eine Gegenüberstellung der mit Hilfe von Einzelmethoden ermittelten Ergebnisse (beispielsweise durch sog. Joint-Displays; Creswell und Plano Clark 2018) auch darüberhinausgehende, als Meta-Inferenzen bezeichnete Befunde herausgearbeitet werden müssen. Diese sind

gebündelte Schlussfolgerungen höherer Ordnung [...], die darauf abzielen, gemeinsame Schlussfolgerungen aus den sich bestätigenden, widersprechenden

\footnotetext{
5 Als Beispiel sei etwa auf Bartolini-Bussi (1994) verwiesen, die die unvermeidliche Notwendigkeit des Gebrauchs verschiedener theoretischer Perspektiven (hier Piaget und Vygotski) zur Erschließung von Interaktionen im Mathematikunterricht diskutiert.
} 


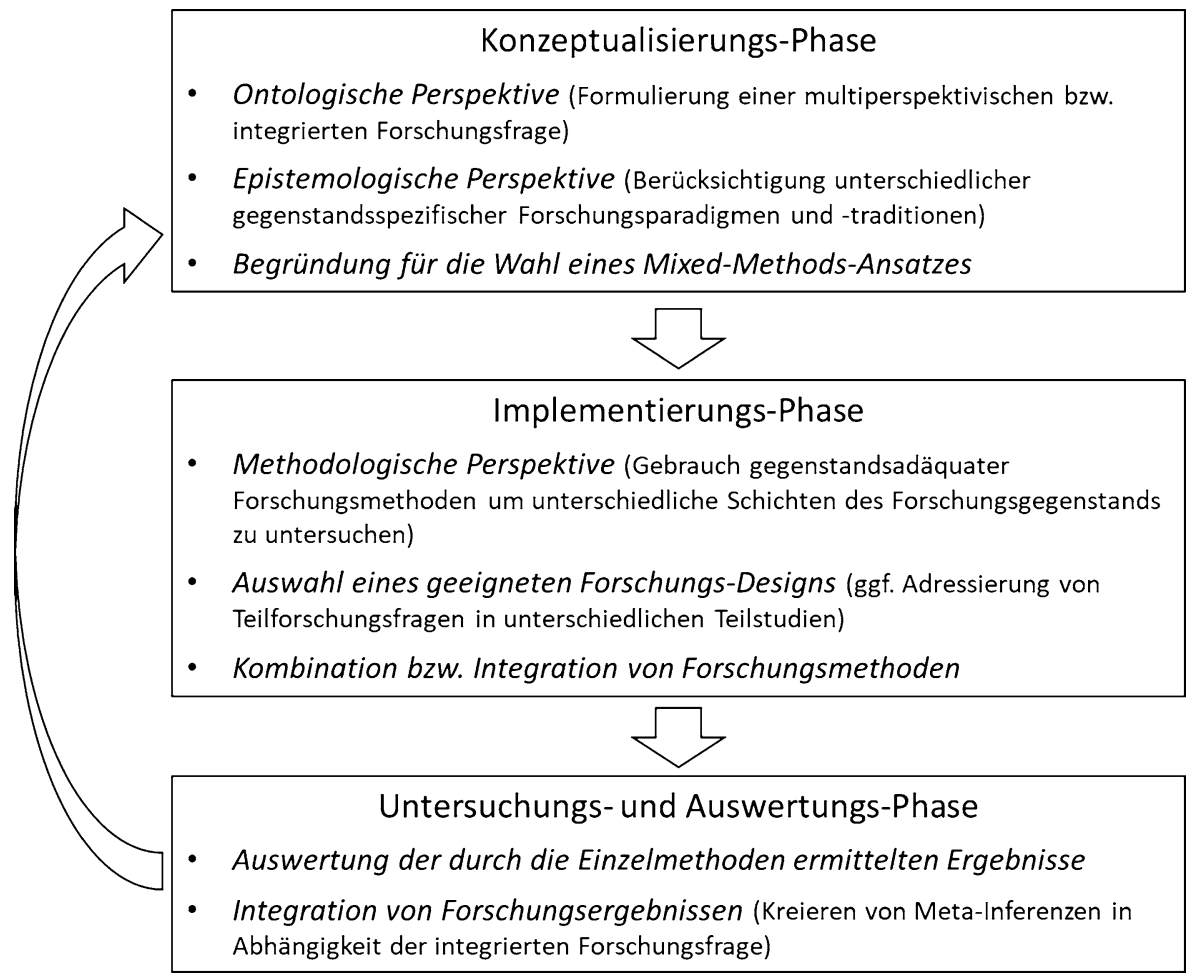

Abb. 1 Framework für den Mixed-Methods-Forschungsansatz (basierend auf Riazi 2016, S. 39)

und ergänzenden Ergebnissen zu ziehen und im gesamten Forschungskontext zu reflektieren. (Hense und Schork 2017, S. 381)

Insbesondere kann bei der Kombination stoffdidaktischer und empirischer Forschungsmethoden der Mehrwert für die mathematikdidaktische Forschung in einer Rückkopplung empirischer Forschungsergebnisse auf die zuvor vorgenommene fachdidaktische Strukturierung von mathematischen Lerninhalten gesehen werden (vgl. etwa Hußmann und Prediger 2016). ${ }^{6}$

Für die Sprachdidaktik hat Riazi (2016) anhand ähnlicher Überlegungen zu Qualitätskriterien ein Framework für Mixed-Methods-Studien entwickelt, das er als „Mixed-Methods-Methodologie“ (S. 38) begreift, und das sich unter Berücksichtigung der oben genannten Kriterien mit Blick auf die mathematikdidaktische Forschung übertragen lässt (vgl. Abb. 1).

Eine Orientierung mathematikdidaktischer Mixed-Methods-Studien an diesem Framework kann die angesprochenen Qualitätskriterien in allen Phasen des Forschungsprozesses berücksichtigen. Dabei sollte der Forschungsprozess mit Blick

\footnotetext{
${ }^{6}$ Der Ordnung halber sei erwähnt, dass die in Hußmann und Prediger (2016) angelegte Studie nicht als Mixed-Methods-Studie angelegt bzw. deklariert ist (mdl. komm. mit S. Hußmann).
} 
auf die beschriebenen Qualitätskriterien durch folgende Fragen strukturiert werden (vgl. Buchholtz 2019):

Konzeptualisierungs-Phase:

- Inwieweit erweist sich mathematisches Wissen im Bildungszusammenhang einer Untersuchung zugänglich?

- Welche Formen von Mehrschichtigkeit weist der Forschungsgegenstand auf? Welche Aspekte des Forschungsgegenstandes sollen untersucht werden?

- Weist der Forschungsgegenstand verschiedene konstituierende Aspekte auf, wie z. B. Akteure auf verschiedenen Ebenen (z. B. Lehrende und Studierende oder individuelle und institutionelle Bedingungen)? Hat der Forschungsgegenstand eine theoretische und/oder praktische Ausrichtung?

- Welche übergreifende Forschungsfrage kann in Bezug auf den Forschungsgegenstand formuliert werden? Wie lauten spezifische Teilforschungsfragen in Bezug auf einzelne Schichten?

- Aus welchen epistemologischen Perspektiven lassen sich unterschiedliche Schichten des Forschungsgegenstandes untersuchen?

- Welcher Mehrwert ergibt sich durch eine Kombination bzw. durch eine Integration unterschiedlicher Methoden?

Implementierungs-Phase:

- Welche Methoden eignen sich zur Beantwortung der Teilforschungsfragen?

- Für welche Forschungsfragen werden qualitative oder quantitative Methoden benötigt? Erfordert die Untersuchung des Forschungsgegenstandes die Anwendung stoffdidaktischer Methoden?

- An welchen Stellen kann eine Integration von Methoden stattfinden? Wie kann/ soll die Integration aussehen?

- Welches Forschungsdesign passt zur Fragestellung und zur Begründung der Studie? Wie verhalten sich die einzelnen Komponenten im Forschungsdesign zueinander?

Untersuchungs- und Auswertungs-Phase:

- Welche stoffdidaktischen Erkenntnisse werden erzielt?

- Werden in der Studie qualitative und/oder quantitative Befunde generiert? Werden die zugehörigen Daten unabhängig voneinander erhoben und analysiert oder findet die Integration bereits auf der Ebene der Datenerhebung und -analyse statt?

- Welche Informationen über die verschiedenen Schichten des Forschungsgegenstands halten die Ergebnisse bereit? Wo ergeben sich Anknüpfungspunkte für die Integration der Ergebnisse?

- Welcher ontologische Mehrwert ergibt sich durch eine Integration der Ergebnisse?

- Welche Meta-Inferenzen können formuliert werden?

\section{Abschließende Bemerkungen}

Im Folgenden möchte ich einige Bemerkungen über die methodologischen Voraussetzungen von MMF in der Mathematikdidaktik anschließen und einen Ausblick 
auf methodologische Weiterentwicklungen geben. In Bezug auf die mathematikdidaktische Forschung kann die MMF ebenfalls als „Mitten-Paradigma“ zwischen verschiedenen Epistemologien verstanden werden und es ermöglichen, ontologisch begründet Forschungsmethoden unterschiedlicher epistemologischer Ansätze in der Mathematikdidaktik in Abhängigkeit vom Forschungsgegenstand miteinander zu kombinieren oder zu integrieren. Die theoretische Verankerung von Mixed-MethodsStudien innerhalb des Critical Realism ermöglicht es dabei, einen gegenstandspezifischen Mehrwert der Integration von Forschungsergebnissen zu identifizieren und so rein technischen Parallelausführungen von durch Einzelmethoden ermittelten Ergebnissen vorzubeugen. Dieses Verständnis von MMF sollte in der Mathematikdidaktik allerdings bewusst nicht ,drittes Paradigma“ genannt werden, wie es in der MixedMethods-Community sonst gebräuchlich ist (Johnson und Onwuegbuzie 2004). Das Forschungsfeld der Mathematikdidaktik ist u.a. durch den synergistischen Zusammenhang zwischen den Bezugswissenschaften und der Mathematik geprägt (Bishop 1992) sowie durch einen starken Praxisbezug. Als Mitten-Paradigma nimmt die mathematikdidaktische MMF hier also nicht nur eine Position zwischen qualitativer und quantitativer Forschungsmethodik innerhalb der empirischen Sozialwissenschaften und innerhalb der ihnen unterliegenden Forschungsparadigmen ein, sondern es spielen auch weitere Paradigmen eine Rolle, wie etwa das wissenschaftliche Paradigma der Mathematik mit seinen verschiedenen (und durchaus kontroversen) Philosophien und den zugehörigen Praktiken (Davis und Hersh 1981; Ernest 2018; Sierpinska und Lerman 1996) sowie den daran angeschlossenen stoffdidaktischen Methoden (s. Abschn. 5.2). Das Argument für Mixed Methods, dass sich bei einer Kombination oder Integration unterschiedlicher Forschungsmethoden deren Stärken addieren bzw. deren Schwächen gegenseitig kompensieren, lässt sich auf den Gegenstandsbereich mathematikdidaktischer Forschung übertragen. Allerdings ist hierzu ein erweitertes Verständnis von Forschungsmethoden innerhalb des gemischt-methodischen Vorgehens erforderlich. Dazu kann auf den breiten Integrationsbegriff der Mixed-Methods-Community und auf die Offenheit gegenüber der Kombination und Integration auch unterschiedlicher Forschungsparadigmen verwiesen werden. Diese ermöglichen eine entsprechende Ausweitung des Mixed-Methods-Begriffs auch auf den Gebrauch stoffdidaktisch-orientierter fachbezogener Forschung. In Abhängigkeit vom Forschungsgegenstand kann damit auch die Kombination bzw. Integration empirisch-sozialwissenschaftlicher und stoffdidaktischer Forschungsmethoden erfolgen (Buchholtz 2019). Bislang existieren allerdings noch nicht viele Studien, die eine derartige Kombination unter einer methodologischen Mixed-Methods-Perspektive vornehmen. Aussichtsreich erscheinen in diesem Zusammenhang aber Studien, die an die fachdidaktische Entwicklungsforschung angelehnt sind, da hier sowohl stoffdidaktische als auch empirische Perspektiven miteinander vereint werden. Es ergeben sich aus methodologischer Sicht aber auch die weiterführenden Fragen, ob und wie sich existierende mathematikdidaktische Mixed-Methods-Studien klassifizieren lassen, wie in verschiedenen Klassen eine Integration von Forschungsergebnissen aussehen kann und auf welche Arten von Meta-Inferenzen geschlossen werden kann. Hier könnte eine mathematikdidaktisch motivierte Prävalenz-Studie aufschlussreich sein, die u. a. auch disziplinspezifische Begründungen von MixedMethods-Studien identifizieren könnte. Einschränkend ist zu sagen, dass nicht je- 
de mathematikdidaktische Studie, in der unterschiedliche Forschungsmethoden zum Einsatz kommen, zwangsläufig eine Mixed-Methods-Studie ist. Die Wahl der methodologischen Verortung innerhalb dieses Forschungsansatzes sollte im Einzelfall immer unter der Berücksichtigung des Forschungs- und Erkenntnisinteresses und der jeweiligen Forschungsfrage abgewogen werden. Wenn eine mathematikdidaktische Studie allerdings innerhalb dieses Forschungsansatzes verortet wird, sollte sie über entsprechende Qualitätskriterien verfügen, zu denen hier erste disziplinspezifische Ansätze entwickelt wurden.

Danksagung Ich möchte den Gutachterinnen und Gutachtern des Beitrags für das JMD meinen herzlichen Dank aussprechen. Der Beitrag hat von den kritisch-konstruktiven Rückmeldungen stark profitiert und durch die entsprechenden Überarbeitungen deutlich an Form gewonnen. Mein Dank für entscheidende Impulse zu gegebener Zeit gilt außerdem Burke Johnson, Judith Huget, Rita Borromeo-Ferri, Timo Leuders und Armin Jentsch.

Funding Open Access funding provided by University of Oslo (incl Oslo University Hospital).

Open Access Dieser Artikel wird unter der Creative Commons Namensnennung 4.0 International Lizenz veröffentlicht, welche die Nutzung, Vervielfältigung, Bearbeitung, Verbreitung und Wiedergabe in jeglichem Medium und Format erlaubt, sofern Sie den/die ursprünglichen Autor(en) und die Quelle ordnungsgemäß nennen, einen Link zur Creative Commons Lizenz beifügen und angeben, ob Änderungen vorgenommen wurden.

Die in diesem Artikel enthaltenen Bilder und sonstiges Drittmaterial unterliegen ebenfalls der genannten Creative Commons Lizenz, sofern sich aus der Abbildungslegende nichts anderes ergibt. Sofern das betreffende Material nicht unter der genannten Creative Commons Lizenz steht und die betreffende Handlung nicht nach gesetzlichen Vorschriften erlaubt ist, ist für die oben aufgeführten Weiterverwendungen des Materials die Einwilligung des jeweiligen Rechteinhabers einzuholen.

Weitere Details zur Lizenz entnehmen Sie bitte der Lizenzinformation auf http://creativecommons.org/ licenses/by/4.0/deed.de.

\section{Literatur}

Archer, M., Bhaskar, R., Collier, A., Lawson, T., \& Norrie, A. (Hrsg.). (1998). Critical realism: essential readings. London: Routledge.

Bakker, A., \& Van Eerde, H. A. A. (2015). An introduction to design based research with an example from statistics education. In A. Bikner-Ahsbahs, C. Knipping \& N. Presmeg (Hrsg.), Doing qualitative research: methodology and methods in mathematics education (S. 429-466). Berlin, Heidelberg, New York: Springer.

Bartolini-Bussi, M. G., \& Bazzini, L. (2003). Research, practice and theory in didactics of mathematics: towards dialogue between different fields. Educational Studies in Mathematics, 54(2-3), 203-223.

Bartolini Bussi, M. (1994). Theoretical and empirical approaches to classroom interaction. In R. Biehler, R. Sträßer \& B. Winkelmann (Hrsg.), Didactics of mathematics as a scientific discipline (S. 121-132). Dordrecht: Kluwer.

Baur, N., Kelle, U., \& Kuckartz, U. (2017). Mixed Methods - Stand der Debatte und aktuelle Problemlagen. KZfSS Kölner Zeitschrift für Soziologie und Sozialpsychologie, 69(Supplement 2), 1-37.

Bazeley, P. (2017). Integrating analyses in mixed methods research. London: SAGE.

Beer, B. (2008). „Methode“, „Methodik“ und „Methodologie“ in der Ethnologie. Ethnoscripts, 10(2), $8-23$.

Bhaskar, R. (1989). Reclaiming reality: a critical introduction to contemporary philosophy. London: Verso.

Bhaskar, R. (1978). A realist theory of science. Hassocks: Harvester.

Biehler, R., \& Blum, W. (2016). Didaktisch orientierte Rekonstruktion von Mathematik als Basis von Schulmathematik und Lehrerbildung - Editorial. Journal für Mathematik-Didaktik, 37, 1-4.

Bigalke, H.-G. (1974). Sinn und Bedeutung der Mathematikdidaktik. ZDM. Zentralblatt für Didaktik der Mathematik, 6(3), 109-115. 
Bikner-Ahsbahs, A., Knipping, C., \& Presmeg, N. (Hrsg.). (2015). Approaches to qualitative research in mathematics education. Examples of methodology and methods. Dordrecht: Springer.

Bishop, A. J. (1992). International perspectives on research in mathematics education. In D. A. Grouws (Hrsg.), Handbook of research on mathematics teaching and learning (S. 710-723). New York: Macmillan.

Brousseau, G. (1981). Problèmes de Didactique des Décimaux. Recherches en Didactique des Mathématiques, 7(2), 33-115.

Bryman, A. (2016). Social research methods. Oxford: Oxford University Press.

Bryman, A. (2008). Why do researchers integrate/combine/mesh/blend/mix/merge/fuse quantitative and qualitative research? In M. Bergman (Hrsg.), Advances in mixed method research (S. 87-100). London: SAGE.

Buchholtz, N. (2019). Planning and conducting mixed methods studies in mathematics educational research. In G. Kaiser \& N. Presmeg (Hrsg.), Compendium for early career researchers in mathematics education (ICME-13 monographs) (S. 131-152). Cham: Springer.

Burkhardt, H., \& Schoenfeld, A.H. (2003). Improving educational research: toward a more useful, more influential, and better-funded enterprise. Educational Researcher, 32(9), 3-14.

Cobb, P., Confrey, J., diSessa, A., Lehrer, R., \& Schauble, L. (2003). Design experiments in education research. Educational Researcher, 32(1), 9-13.

Creswell, J. W., \& Plano Clark, V. L. (2018). Designing and conducting mixed methods research. Thousand Oaks: SAGE.

Danermark, B., Ekström, M., \& Karlsson, J.C. (2019). Explaining society. Critical realism in the social sciences. New York: Routledge.

Davis, P., \& Hersh, R. (1981). The mathematical experience. New York: Houghton Mifflin.

Denzin, N. (1978). The research act. A Theoretical Introduction to Sociolological Methods. New York: McGraw-Hill.

Ernest, P. (2018). The philosophy of mathematics education: an overview. In P. Ernest (Hrsg.), The philosophy of mathematics education today. ICME-13 monographs (S. 13-35). Cham: Springer.

Ernest, P. (1998). A postmodern perspective on research in mathematics education. In A. Sierpinska \& J. Kilpatrick (Hrsg.), Mathematics education as a research domain-an ICMI study (Bd. 1-2, S. 71-85). Dordrecht: Kluwer.

Flick, U. (1998). An introduction to qualitative research. Thousand Oaks: SAGE.

Gage, N. (1989). The paradigm wars and their aftermath: a "historical" sketch of research and teaching since 1989. Educational Researcher, 18, 4-10.

Greene, J. C. (2015). Preserving distinctions within the MMR merger. In S. J. N. Hesse-Biber \& R. B. Johnson (Hrsg.), The Oxford handbook of multimethod and mixed methods research inquiry (S. 606-615). New York: Oxford University Press.

Greene, J. C., Caracelli, V. J., \& Graham, W.F. (1989). Toward a conceptual framework for mixed-method evaluation designs. Educational Evaluation and Policy Analysis, 11, 255-274.

Griesel, H. (1997). Zur didaktisch orientierten Sachanalyse des Begriffs Größe. Journal für MathematikDidaktik, 18, 259-284.

Griesel, H. (1974). Überlegungen zur Didaktik der Mathematik als Wissenschaft. Zentralblatt für Didaktik der Mathematik, 6(3), 115-119.

Griesel, H. (1972). Die mathematische Analyse als Forschungsmittel in der Didaktik der Mathematik. In Beiträge zum Mathematikunterricht 1971 - Vorträge auf der 5. Bundestagung für Didaktik der Mathematik vom 14. bis 16. April 1971 in Bayreuth (S. 72-81). Hannover: Schroedel.

Guba, E. G. (1990). The paradigm dialog. Newbury Park: SAGE.

Habermas, J. (1972). Knowledge and human Interests. London: Heinemann.

Hammersley, M. (2002). The relationship between qualitative and quantitative research: paradigm loyality versus methodological eclecticism. In J. Richardson (Hrsg.), Handbook of qualitative research methods for psychology and the social science (S. 159-174). Oxford: BPS Blackwell.

Hart, L.C., Smith, S.Z., Swars, S.L., \& Smith, M.E. (2009). An examination of research methods in mathematics education (1995-2005). Journal of Mixed Methods Research, 3(1), 26-41.

Hense, A., \& Schork, F. (2017). Doing Mixed Methods: Methodenintegrative Ansätze in der Organisationsforschung. In S. Liebig, W. Matiaske \& S. Rosenbohm (Hrsg.), Handbuch Empirische Organisationsforschung (S. 1-30). Wiesbaden: Springer.

Hoffkamp, A. (2014). Stoffdidaktik im Fokus - Das Beispiel Lineare (Un-)Abhängigkeit. In J. Roth \& J. Ames (Hrsg.), Beiträge zum Mathematikunterricht 2014 (S. 543-546). Münster: WTM-Verlag.

Hoth, J. (2016). Situationsbezogene Diagnosekompetenz von Mathematiklehrkräften. Eine Vertiefungsstudie zur TEDS-Follow-Up-Studie. Wiesbaden: Springer. 
Howe, K. (1988). Against the quantitative-qualitative incompatibility thesis, or dogmas die hard. Educational Researcher, 17, 10-16.

Hußmann, S., \& Prediger, S. (2016). Specifying and structuring mathematical topics-a four-level approach for combining formal, semantic, concrete, and empirical levels exemplified for exponential growth. Journal für Mathematik-Didaktik, 37(1), 33-67.

Johnson, R.B., \& Christensen, L. B. (2017). Educational research: Quantitative, qualitative, and mixed approaches. Thousand Oaks: SAGE.

Johnson, R. B., Onwuegbuzie, A. J., \& Turner, L. A. (2007). Toward a definition of mixed methods research. Journal of Mixed Methods Research, 1(2), 112-133.

Johnson, R. B., \& Onwuegbuzie, A. J. (2004). Mixed methods research: a research paradigm whose time has come. Educational Researcher, 33(7), 14-26.

Kelle, U. (2017). Die Integration qualitativer und quantitativer Forschung - theoretische Grundlagen von „Mixed Methods“. KZfSS Kölner Zeitschrift für Soziologie und Sozialpsychologie, 69(Supplement 2), 39-61.

Kelle, U., \& Buchholtz, N. (2015). The combination of qualitative and quantitative research methods in mathematics education: a "mixed methods" study on the development of the professional knowledge of teachers. In A. Bikner-Ahsbahs, C. Knipping \& N. Presmeg (Hrsg.), Approaches to qualitative research in mathematics education (S. 321-361). Dordrecht: Springer.

Kirsch, A. (1969). Eine Analyse der sogenannten Schlußrechnung. Mathematisch-Physikalische Semesterberichte, 16(1), 41-55.

Kirsch, A. (1977). Aspects of simplification in mathematics teaching. In H. Athen \& H. Kunle (Hrsg.), Proceedings of the third international congress on mathematical education (S. 98-119).

Kuckarts, U. (2014). Mixed Methods. Methodologie, Forschungsdesigns und Analyseverfahren. Wiesbaden: Springer VS.

Lakoff, G. (1987). Women, fire, and dangerous things: what categories reveal about the mind. Chicago: University of Chicago Press.

Lambert, A. (2014). Teilprozesse der stoffdidaktischen Methode (Poster). Mitteilungen der Gesellschaft für Didaktik der Mathematik, 96, 89.

Lüken, M., \& Sauzet, O. (2020). Patterning strategies in early childhood: a mixed methods study examining 3- to 5-year-old children's patterning competencies. Mathematical Thinking and Learning. https:// doi.org/10.1080/10986065.2020.1719452.

Maxwell, J. A., \& Mittapalli, K. (2010). Realism as a stance for mixed methods research: linking theory to practice. In A. Tashakkori \& C. Teddlie (Hrsg.), Sage handbook of mixed methods in social \& behavioral science (S. 145-167). Thousand Oaks: SAGE.

Mertens, D.M., Bazeley, P., Bowleg, L., Fielding, N., Maxwell, J., Molina-Azorin, J.F., \& Niglas, K. (2016). MMIRA task force report. The future of mixed methods: a five year projection to 2020. https://mmira.wildapricot.org/resources/Documents/MMIRA\%20task\%20force\%20report \%20Jan2016\%20final.pdf. Zugegriffen: 09.09.2020

Mittelstraß, J. (Hrsg.). (2013). Log-N. Enzyklopädie Philosophie und Wissenschaftstheorie, Bd. 5. Berlin, Heidelberg, New York: Springer.

Morgan, D.L. (2018). Living within blurry boundaries: the value of distinguishing between qualitative and quantitative research. Journal of Mixed Methods Research, 12(3), 268-279.

Morgan, D. L. (2007). Paradigms lost and pragmatism regained: methodological implications of combining qualitative and quantitative methods. Journal of Mixed Methods Research, 1(1), 48-76.

Newton, P.E., \& Shaw, S. D. (2014). Validity in educational and psychological assessment. London: SAGE.

Otte, M. (1982). Fachdidaktik als Wissenschaft. Occasional Paper, Bd. 20. IDM Bielefeld

Prediger, S., Link, M., Hinz, R., Hußmann, S., Thiele, J., \& Ralle, B. (2012). Lehr-Lernprozesse initiieren und erforschen-Fachdidaktische Entwicklungsforschung im Dortmunder Modell. $M N U, 65(8)$, 452-457.

Reindl, S. (2016). Lösungsstrategien Addition und Subtraktion. Eine Studie zur Nutzung und Wirkung im Grundschulalter. Münster: Waxmann.

Riazi, A.M. (2016). Innovative mixed-methods research: moving beyond design technicalities to epistemological and methodological realizations. Applied Linguistics, 37(1), 33-49.

Riazi, A. M., \& Candlin, C. N. (2014). Mixed-methods research in language teaching and learning: opportunities, issues and challenges. Language Teaching, 47(2), 135-173.

Ross, A., \& Onwuegbuzie, A. J. (2012). Prevalence of mixed methods research in mathematics education. The Mathematics Educator, 22(1), 84-113.

Salzman, P.C. (2006). Methodology. In A. Barnard \& J. Spencer (Hrsg.), Encyclopedia of social and cultural anthropology (S. 364-367). London, New York: Routledge. 
Scheiner, T. (2019). If we want to get ahead, we should transcend dualisms and foster paradigm pluralism. In G. Kaiser \& N. Presmeg (Hrsg.), Compendium for early career researchers in mathematics education (ICME-13 monographs) (S. 511-532). Cham: Springer.

Schoenfeld, A.H. (2016). Research in mathematics education. Review of Research in Education, 40(1), 497-528.

Schoenfeld, A.H. (2002). Research methods in (mathematics) education. In L. D. English (Hrsg.), Handbook of international research in mathematics education (S. 435-487). Mahwah: Lawrence Erlbaum.

Schoonenboom, J., \& Johnson, J. B. (2017). How to construct a mixed methods research design. KZfSS Kölner Zeitschrift für Soziologie und Sozialpsychologie, 69(Supplement 2), 107-131.

Schüler-Meyer, A., Prediger, S., Kuzu, T., Wessel, L., \& Redder, A. (2017). Is formal language proficiency in the home language required to profit from a bilingual teaching intervention in mathematics? A mixed methods study on fostering multilingual students' conceptual understanding. International Journal for Science and Mathematics Education, 17(2), 317-339.

Schulz, A. (2010). Ergebnisorientierung als Chance für den Mathematikunterricht? Innovationsprozesse qualitativ und quantitativ erfassen. München: Utz Verlag.

Sierpinska, A., \& Kilpatrick, J. (Hrsg.). (1998). Mathematics education as a research domain-an ICMI study. Bd. 1-2. Dordrecht: Kluwers.

Sierpinska, A., \& Lerman, S. (1996). Epistemologies of mathematics and of mathematics education. In A. J. Bishop, et al. (Hrsg.), International handbook of mathematics education (Bd. 1-2, S. 827-876). Dordrecht: Kluwer.

Steinbring, H. (2011). Changed views on mathematical knowledge in the course of didactical theory development: independent corpus of scientific knowledge or result of social constructions? In T. Rowland \& K. Ruthven (Hrsg.), Mathematical knowledge in teaching (S. 43-64). Berlin: Springer.

Steinbring, H. (1998). Mathematikdidaktik: Die Erforschung theoretischen Wissens in sozialen Kontexten des Lernens und Lehrens. ZDM. Zentralblatt für Didaktik der Mathematik, 30(5), 161-167.

Steiner, H.-G. (1985). Theorie der Mathematikdidaktik. Hintergründe und Informationen zu einem auf dem 5. Internationalen Kongreß für Mathematikdidaktik 1984 in Adelaide, Australien, begonnenen Programm. Zentralblatt für Didaktik der Mathematik, 17(2), 57-65.

Sträßer, R. (2015). Mathematikdidaktik - mehr als das Design praktikabler Kurse für den Mathematikunterricht. Eine Replik auf Erich C. Wittmann. Mitteilungen der Gesellschaft für Didaktik der Mathematik, 99, 30-32.

Tashakkori, A., \& Teddlie, C. (2003). Handbook of mixed methods in social \& behavioral research. Thousand Oaks: SAGE.

van den Akker, J., Gravemeijer, K., McKenney, S., \& Nieveen, N. (Hrsg.). (2006). Educational design research. London: Routledge.

Vohns, A. (2007). Grundlegende Ideen und Mathematikunterricht. Entwicklung und Perspektiven einer fachdidaktischen Kategorie. Norderstedt: Books on Demand.

Winter, H. (1985). Reduktionistische Ansätze in der Mathematikdidaktik. Der Mathematikunterricht (MU), 31(5), 75-88.

Wittmann, E. C. (1992). Mathematikdidaktik als „,design science“. Journal für Mathematik-Didaktik, 13(1), 55-70. 